\section{Experiencia de 22 años de trasplante autólogo de células hematopoyéticas en pacientes con mieloma múltiple o amiloidosis sistémica. 1992-2014}

\author{
MAURICIO SARMIENTO, PABLO LIRA, MAURICIO OCQUETEAU, \\ MARÍA ALEJANDRA RODRÍGUEZ, MARÍA JOSÉ GARCÍA, \\ VERÓNICA JARA, PABLO BERTÍN, PABLO RAMÍREZ
}

\section{Autologous hematopoietic cell transplantation in patients with multiple myeloma. Experience in 53 patients}

Background: Autologous hematopoietic cell transplantation (THA) in patients with multiple myeloma and amyloidosis is the standard of care to promote disease free survival and quality of life. Aim: To report our experience with THA in patients with multiple myeloma. Material and Methods: Retrospective review of the hematopoietic cell transplantation database of a hospital of a Medical School. Forty seven patients with multiple myeloma and six with amyloid light chain amyloidosis were identified. Clinical and demographic data were obtained from the records. Results: The overall five year survival of patients was 55\%. Transplant-related or non-relapse mortality occurred in 7\%. We found no differences in outcomes among patients younger or older than 50 years. Conclusions: Our data supports that THA can be done in our country with similar results to those obtained in international transplantation centers. Chronological age should not be a limitation to offer this therapy to patients with multiple myeloma and amyloidosis.

(Rev Med Chile 2014; 142: 1497-1501)

Key words: Amyloidosis; Hematopoietic stem cell transplantation; Multiple myeloma.

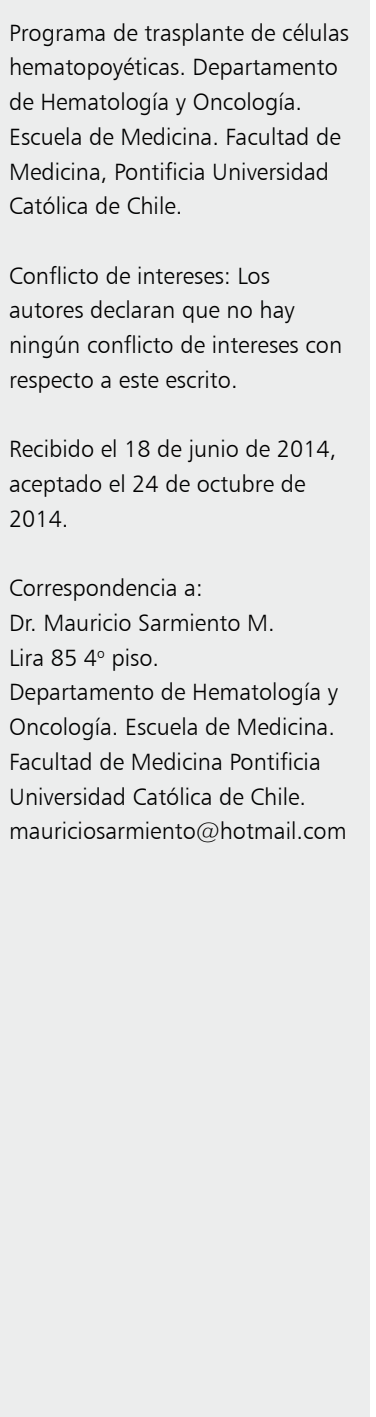

4 1 trasplante autólogo de células hematopoyéticas (TAH) es considerado el estándar de tratamiento para pacientes jóvenes (menores de 70 años) con mieloma múltiple (MM) o gammopatías graves como la amiloidosis. Si bien el objetivo del TAH en MM no es la curación de la enfermedad, existe evidencia que muestra una mejor sobrevida libre de progresión y de eventos y mejor calidad de vida, con períodos libres de tratamiento que en aquellos tratados sólo con quimioterapia ${ }^{1-3}$. Los resultados obtenidos en estos estudios mues- tran sobrevida global a 5 años de $60 \%$ y sobrevida libre de progresión de 31 meses, con mortalidad precoz asociada al trasplante cercana a $4 \%^{1-3}$.

Nuestra Red de Salud Universidad Católica de Chile tiene experiencia en el manejo de pacientes con enfermedades oncológicas graves y trasplante de diversos órganos, incluyendo el trasplante de progenitores hematopoyéticos ${ }^{4}$. En este reporte mostramos nuestra experiencia en los últimos 22 años en pacientes con MM o amiloidosis que recibieron TAH. 


\section{Materiales y Métodos}

Se realizó un análisis retrospectivo de la base de datos del programa de trasplante de células hematopoyéticas de nuestra facultad de medicina. Desde 1992 hasta 2014 se han realizado 358 trasplantes en adultos, de los cuales 53 correspondieron a TAH en pacientes con gammopatias, 47 con MM y 6 con amiloidosis AL. Se recopilaron datos demográficos y específicos de la enfermedad tales como fecha de diagnóstico, subtipo del mieloma, estado de la enfermedad al momento del trasplante, fecha del trasplante, cantidad de células CD34 infundidas, tipo de condicionamiento, días de prendimiento de neutrófilos y plaquetas, complicaciones, causa de muerte, si la hubo, y tiempo de sobrevida libre de enfermedad.

El status de la enfermedad se estableció de acuerdo a los criterios del Myeloma Working Group ${ }^{5}$. Brevemente, la remisión completa (RC) se estableció por la ausencia de peak monoclonal e inmunofijación en sangre periférica y orina y presencia de menos de $10 \%$ de células plasmáticas normales en médula ósea. Muy buena respuesta parcial (MBRP) se estableció por los mismos criterios de la RC, salvo que había persistencia de inmunofijación positiva en sangre $u$ orina. La remisión parcial $(\mathrm{RP})$ se estableció por la presencia de disminución parcial del clon de mielomatosis en médula sin lograr valores normales con disminución, más no resolución del peak monoclonal y enfermedad activa se definió en aquellos pacientes que no presentaron respuesta alguna al tratamiento.

La enfermedad medular al diagnóstico y al seguimiento se evaluó de dos maneras: con mielograma, en donde a la microscopia convencional se observó la cantidad y aspecto morfológico de la hematopoyesis y donde se definió la presencia de plasmoblastos con un corte de más de 10\% como indicador diagnóstico de MM y por medio de inmunofenotipo determinado por citometría de flujo, donde se evaluaron clusters de diferenciación (CD), estableciendo la presencia de MM [>95\% de plasmoblastos con inmunofenotipo CD19 (-) CD56 (+) CD45 (-) CD38 (+) y restricción de cadenas livianas kappa o lambda intracitoplasmática] y de células plasmáticas normales [CD19 (+) CD56 (-) CD45 (+) CD38 $(++)$ con expresión policlonal de cadenas livianas kappa y lambda].
La cosecha de la médula ósea se realizó con estimulación con filgrastim, a dosis de $600 \mathrm{mcg}$ subcutáneos cada día, por 5 días, seguido de proceso de leucoaferesis por vía periférica. En aquellos que no lograron recuentos satisfactorios con la primera aféresis (por lo menos 2,5 x $10^{6} \mathrm{CD} 34$ / $\mathrm{kg}$ ) se realizaron o nuevas cosechas en un período de tres semanas o se usó plerixafor.

Se realizó análisis estadístico con test de Gehan-Breslow-Wilcoxon para las estimaciones de sobrevida y se utilizaron cálculos de medias aritméticas. La diferencia estadísticamente significativa se definió con valores de $\mathrm{p}<0,05$. Las curvas de sobrevida fueron realizadas con Graphpad Prism versión 6 . Los resultados se midieron de acuerdo a los consensos del EBMT; brevemente, la sobrevida global se definió como el tiempo transcurrido hasta la muerte, independiente de la causa, y la mortalidad no asociada a recaída-asociada a TMO se definió como cualquier causa de muerte sin presencia de recaída o recidiva del $\mathrm{MM}^{6}$.

\section{Resultados}

\section{Características de los pacientes}

En los últimos 22 años, realizamos 53 trasplantes autólogos en MM. En la Tabla 1 se describen las características de los pacientes. En suma, el promedio de edad fue de 53 años (rango 37-67), en su mayoría hombres y principalmente con subtipo IgG. En los primeros años del programa se realizó condicionamiento con busulfan, melfalán y tiotepa. Luego, debido a la evidente toxicidad observada en nuestros pacientes y en base a evidencia ${ }^{7}$, se cambió a melfalán con dosis estándar o bajas, dependiendo de la presencia o no de falla renal.

En relación al subtipo del mieloma, la Tabla 2 muestra que de los 53 pacientes, 31 eran portadores de MM IgG, 5 de MM IgA, 4 de MM de cadenas livianas, 2 de MM no secretores y 6 pacientes eran portadores de amiloidosis sistémica tipo AL. En 5 pacientes no se determinó el subtipo.

\section{Status de la enfermedad al momento del trasplante (Tabla 3)}

De los 53 pacientes, 34 llegaron al trasplante en primera remisión completa, 8 en muy buena respuesta parcial, 1 en remisión parcial, 9 con enfermedad activa y 1 en segunda remisión completa. 
Trasplante autólogo de células hematopoyéticas en mieloma múltiple - M. Sarmiento et al

Tabla 1. Características de los pacientes

\begin{tabular}{|c|c|}
\hline Pacientes & $n=53$ \\
\hline $\begin{array}{l}\text { Edad (rango) } \\
>50 \text { años } \\
<50 \text { años }\end{array}$ & $\begin{array}{l}\text { Promedio } 53 \text { años }(37-69) \\
n=33 \text { promedio } 57(51-69) \\
n=20 \text { promedio } 44(37-49)\end{array}$ \\
\hline Relación masculino/femenino & $27 / 26$ \\
\hline Falla renal al trasplante (Clearance creatinina menor a $10 \mathrm{ml} / \mathrm{min}$ ) & $6(12 \%)$ \\
\hline $\begin{array}{l}\text { Tipo de quimioterapia condicionante usado } \\
\text { - Busulfan-melfalán-tiotepa } \\
\text { - Melfalán } 200 \mathrm{mg} / \mathrm{m}^{2} \\
\text { - Melfalán } 140 \mathrm{mg} / \mathrm{m}^{2}\end{array}$ & $\begin{array}{r}10 \\
37 \\
6\end{array}$ \\
\hline
\end{tabular}

Tabla 2. Subtipos de gammopatías

\begin{tabular}{|lc|}
\hline Pacientes & $\mathrm{n}=53$ \\
MM IgG & 31 \\
- IA & 9 \\
- IIA & 7 \\
- IIIA & 11 \\
MM IIIB & 4 \\
- IIA & 5 \\
- IIIA & 2 \\
- IIIB & 2 \\
MM cadenas livianas & 1 \\
Amiloidosis sistémica AL & 4 \\
No secretor & 6 \\
No determinado & 2 \\
\hline
\end{tabular}

Los tratamientos de quimioterapias utilizadas para llevar a la remisión completa variaron de acuerdo a la fecha de tratamiento. De esta manera, 6 pacientes tratados antes del año 2000 recibieron esquema VAD (vincristina, adriamicina, dexametasona) o MPT (melfalán, prednisona, talidomida); 23 pacientes tratados entre el 2001 y 2008 recibieron esquema TD (talidomida y dexametasona) o VAD y 24 pacientes tratados posterior al recibieron esquema VCD (bortezomib, dexametasona, ciclofosfamida). Todos los pacientes que se trasplantaron por amiloidosis tuvieron compromiso renal y ninguno compromiso cardíaco o de otros parénquimas. En su mayoría (90\%) tuvieron creatinina menor a $2 \mathrm{mg} \%$ y proteinuria nefrótica como principal manifestación.

La dosis promedio de CD34 infundida fue
Tabla 3. Status de la enfermedad al momento del trasplante

\begin{tabular}{|lc|}
\hline Pacientes & $\mathrm{n}=53$ \\
CR1 (remisión completa 1) & 34 \\
MBRP (muy buena remisión parcial) & 8 \\
RP (remisión parcial) & 1 \\
Enfermedad activa & 9 \\
CR2 (2 ${ }^{\text {da }}$ remisión completa $)$ & 1 \\
\hline
\end{tabular}

Tabla 4. Características del trasplante

\begin{tabular}{|ll|}
\hline Pacientes & $\mathrm{n}=53$ \\
$\begin{array}{l}\text { Cosecha de células } \\
\text { hematopoyéticas } \\
\text { (medular/sangre periférica) }\end{array}$ & $3 / 50$ \\
$\begin{array}{l}\text { Dosis de células hematopoyéticas } \\
\text { CD34 x 10\%/kg (rango) }\end{array}$ \\
$\begin{array}{l}\text { Movilización } \\
\text { - G-CSF } \\
\text { - G-CSF + plerixafor }\end{array}$ \\
$\begin{array}{l}\text { Prendimiento } \\
\text { - Neutrófilos }\end{array}$ \\
- Plaquetario & 58 \\
\hline
\end{tabular}

de $3,8 \times 10^{6} / \mathrm{kg}$ (rango 1,98-10,1), que fueron obtenidas de un promedio de 1,4 cosechas. Fue necesario utilizar plerixafor como adyuvante en la recolección en 5 pacientes, que resultaron ser malos movilizadores.

Se evidenció mucositis grado 1 ó 2 en todos los pacientes. En $70 \%(n=37)$ se presentó neutrope- 


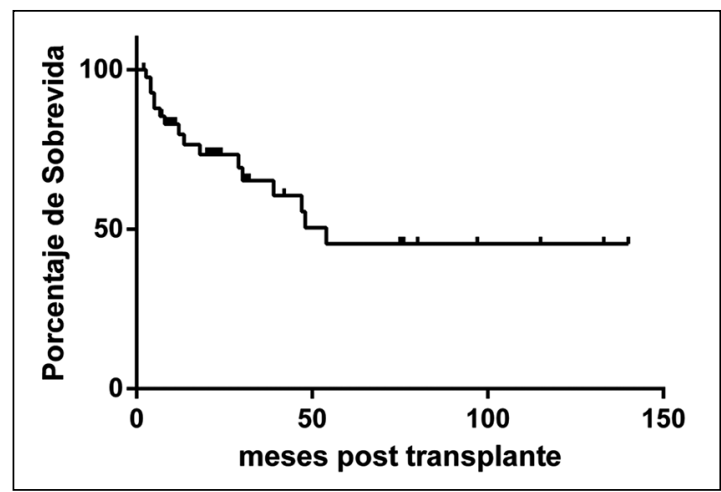

Figura 1. Sobrevida global.

nia febril con respuesta adecuada al tratamiento antibiótico. El prendimiento de los neutrófilos se logró en promedio el día 11 post infusión. (rango: 10-26). El prendimiento plaquetario (recuento mayor a 20.000 plaquetas/uL por 5 días consecutivos) se alcanzó en promedio, el día 13 (rango: 8-36) (Tabla 4).

\section{Sobrevida global y mortalidad}

Con una media de seguimiento de 5 años, la sobrevida global fue $55 \%$, observando algunos pacientes $(\mathrm{n}=22)$ con sobrevida prolongada (entre 3 y 8 años) (Figura 1). Diecisiete (32\%) pacientes han fallecido. La mortalidad por trasplante (no asociada a recaída) se concentró en los primeros 10 años del programa, cuando se usó condicionamiento de máxima intensidad; entre el año 1992 y el año 2000 hubo 10 pacientes trasplantados y 9 fallecidos, lo que se traduce en $90 \%$ de la mortalidad global. Después del año 2001 se modificó el protocolo de condicionamiento, se realizaron 43 trasplantes y hubo 7 fallecidos, dando una mortalidad para este período de $16 \%(\mathrm{p}<0,01)$, dentro de los cuales, $3(7 \%)$ tuvieron como causa directa de su muerte toxicidad relacionada al trasplante (no asociada a recaída) y 4 fallecieron por progresión de su enfermedad.

No se encontraron diferencias significativas en cuanto a sobrevida al comparar los trasplantados mayores de 50 años $(\mathrm{n}=33$, edad promedio 57 años, rango 51-69) con los menores de 50 años $(n=20$, edad promedio 44 años, rango 37-49) (Figura 2).

Los pacientes que recayeron tuvieron una sobrevida libre de enfermedad desde el trasplante

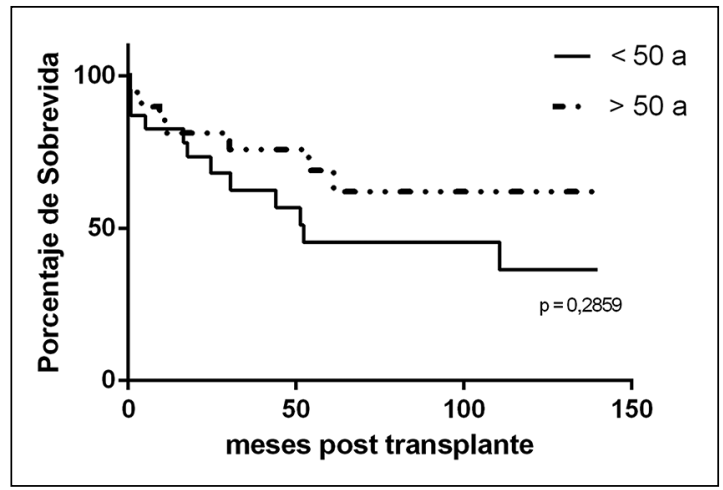

Figura 2. Sobrevida global en mayores y menores de 50 años.

hasta la muerte de 26,8 meses (rango de 4 a 51 meses) (Figura 4).

La sobrevida media de los 6 pacientes con amiloidosis sistémica fue de $50 \%$ a 54 meses, sin diferencias significativas al compararse con el grupo de pacientes trasplantados portadores de mieloma. En este grupo de pacientes se observó 2 muertes por progresión de la enfermedad, a los 4 meses y 39 meses, respectivamente.

\section{Discusión}

El trasplante autólogo de células hematopoyéticas es una estrategia eficaz para mejorar la sobrevida libre de enfermedad de los enfermos con mieloma múltiple. Estudios publicados por centros de tratamiento de mieloma han mostrado que la sobrevida libre de enfermedad y de eventos a 5 años es cercana a $65 \%$ a 5 años de seguimiento $^{1-3,7-11}$. Nuestra experiencia es la más extensa en trasplante autólogo en mieloma múltiple publicada en Chile, mostrando que es una estrategia viable, con toxicidad razonable y con tiempos de sobrevida y mortalidad comparables con centros de referencia internacionales. Es destacable que en nuestra serie, no se observó diferencias en cuanto a los resultados logrados entre pacientes mayores de 50 años. La mayor mortalidad encontrada en nuestra serie, se vio influida por la elevada toxicidad asociada al acondicionamiento en los inicios del programa; una vez que se optimizó, la morbilidad y mortalidad se estabilizaron en las cifras reportadas por otros grupos ${ }^{7}$.

En nuestro país existe información disponible 
Trasplante autólogo de células hematopoyéticas en mieloma múltiple - M. Sarmiento et al

sobre el trasplante en mieloma. Puga y cols. ${ }^{12} \mathrm{pu}-$ blicaron su experiencia en 10 enfermos menores de 40 años, trasplantados, de los cuales 3 tenían MM y tuvieron seguimiento muy breve con respuesta satisfactoria. Flores y cols. ${ }^{13}$ publicaron su experiencia en 20 pacientes con MM que recibieron TAH logrando sobrevida global de $47 \%$.

Nuestros resultados muestran que es posible realizar TAH a pacientes portadores de MM o amiloidosis en nuestro medio y que no debiera haber un límite de edad dado que pacientes menores y mayores de 50 años evolucionan de manera similar.

\section{Referencias}

1. Child JA, Morgan GJ, Davies FE, Owen RG, Bell SE, Hawkins K, et al. High-dose chemotherapy with hematopoietic stem-cell rescue for multiple myeloma. N Engl J Med 2003; 348 (19): 1875.

2. Attal M, Harousseau J, Stoppa A, Sotto J, Fuzibet J, Rossi $\mathrm{J}$, et al. A prospective, randomized trial of autologous bone marrow transplantation and chemotherapy in multiple myeloma. N Engl J Med 1996; 335: 91-7.

3. Palumbo A, Bringhen S, Bruno B, Falcone AP, Liberati AM, Grasso M, et al. Melphalan $200 \mathrm{mg} / \mathrm{m}$ (2) versus melphalan $100 \mathrm{mg} / \mathrm{m}$ (2) in newly diagnosed myeloma patients: a prospective, multicenter phase 3 study. Blood 2010; 115 (10): 1873.

4. Ramírez P, Nervi B, Bertin P, Poggi H, Lagos M, Selman $\mathrm{C}$, et al. Umbilical cord blood transplantation in hematologic diseases in patients over 15 years old: long-term experience at the Pontificia Universidad Católica de Chile. Transplant Proc 2013; 45 (10): 3734-9.

5. Durie BG, Harousseau JL, Miguel JS, et al. International uniform response criteria for multiple myeloma. Leukemia 2006; 20: 1467.

6. Labopin M, Latouche A, Suciu S, Santucci A, Canals C, Iacobelli S, et al. Definitions and evaluation of endpoints following stem cells transplantation - Recommendation from the European group for Blood and Marrow transplantation (EBMT). CLINT, Work package 5. En página web: https://portal.ebmt.org/sites/clint2/clint/ Documents/Statistical\%20Endpoints_CLINT\%20Project_final\%20version.pdf.

7. Martino M, Morabito F, Console G, Irrera G, Messina G, Pucci G, et al. Differences in transplant-related complications between hematologic malignancies and solid tumors receiving high-dose chemotherapy and autologous peripheral blood stem cell transplantation. Tumori 2003; 89 (4): 385-90.

8. Fermand JP, Ravaud P, Chevret S, Divine M, Leblond $\mathrm{V}$, Belanger $\mathrm{C}$, et al. High-dose therapy and autologous peripheral blood stem cell transplantation in multiple myeloma: up-front or rescue treatment? Results of a multicenter sequential randomized clinical trial. Blood 1998; 92 (9): 3131.

9. Elice F, Raimondi R, Tosetto A, D'Emilio A, Di Bona E, Piccin A, et al. Prolonged overall survival with second on-demand autologous transplant in multiple myeloma. Am J Hematol 2006; 81 (6): 426.

10. Anagnostopoulos A, Aleman A, Ayers G, Donato M, Champlin R, Weber D, et al. Comparison of high-dose melphalan with a more intensive regimen of thiotepa, busulfan, and cyclophosphamide for patients with multiple myeloma. Cancer 2004; 100 (12): 2607.

11. Crusoe Ede Q, Higashi F, Padilha MP, Miranda EC, Quero AA, Almeida $\mathrm{M}$ de S, et al. Outcomes of autologous transplantation for multiple myeloma according to different induction regimens. Rev Bras Hematol Hemoter 2014; 36 (1): 19-24.

12. Puga B, Molina J, Andrade A, Guerra C, Ardila A, Álvarez G. [First Ten hematopoietic stem cell transplants performed in the adult public health service in Chile]. Rev Med Chile 2012; 140 (9): 1207-12.

13. Flores C, Conte G, Fardella P, Araos D, Alfaro J, Aravena $\mathrm{P}$, et al. [Autologous transplant (AT) with peripheralblood stem-cell rescue for multiple myeloma. A clinical experience]. Rev Med Chile 2005; 133 (8): 887-93. 